\title{
Ambient-temperature high-pressure-induced ferroelectric phase transition in $\mathrm{CaMnTi}_{2} \mathrm{O}_{6}$
}

\author{
J. Ruiz-Fuertes, ${ }^{1,}$ T. Bernert, ${ }^{2}$ D. Zimmer, ${ }^{3}$ N. Schrodt,${ }^{3}$ M. Koch-Müller, ${ }^{4}$ B. Winkler, ${ }^{3}$ L. Bayarjargal, ${ }^{3}$ C. Popescu, ${ }^{5}$ \\ S. MacLeod, ${ }^{6,7}$ and K. Glazyrin ${ }^{8}$ \\ ${ }^{1}$ MALTA-Consolider Team, Departament de Física Aplicada-ICMUV, Universitat de València, Dr. Moliner 50, 46100 Burjassot, \\ Valencia, Spain \\ ${ }^{2}$ Max-Planck-Institut für Kohlenforschung, Kaiser-Wilhelm-Platz 1, 45470 Mülheim an der Ruhr, Germany \\ ${ }^{3}$ Institut für Geowissenschaften, Goethe-Universität, Altenhöferallee 1, 60438 Frankfurt am Main, Germany \\ ${ }^{4}$ GFZ Potsdam, Sektion 4.3, Telegrafenberg, 14473 Potsdam, Germany \\ ${ }^{5}$ CELLS-ALBA Synchrotron Light Facility, 08290 Cerdanyola del Vallés, Barcelona, Spain \\ ${ }^{6}$ Atomic Weapons Establishment, Aldermaston, Reading RG7 4PR, United Kingdom \\ ${ }^{7}$ Institute of Shock Physics, Imperial College London, London SW7 2AZ, United Kingdom \\ ${ }^{8}$ Deutsches Elektronen-Synchrotron DESY, Notkestrasse 85, D-22603 Hamburg, Germany
}

(Received 16 May 2017; revised manuscript received 16 August 2017; published 5 September 2017)

\begin{abstract}
The ferroelectric to paraelectric phase transition of multiferroic $\mathrm{CaMnTi}_{2} \mathrm{O}_{6}$ has been investigated at high pressures and ambient temperature by second-harmonic generation (SHG), Raman spectroscopy, and powder and single-crystal $\mathrm{x}$-ray diffraction. We have found that $\mathrm{CaMnTi}_{2} \mathrm{O}_{6}$ undergoes a pressure-induced structural phase transition $\left(\mathrm{P}_{2} \mathrm{mc} \rightarrow \mathrm{P}_{2} / \mathrm{nmc}\right)$ at $\sim 7 \mathrm{GPa}$ to the same paraelectric structure found at ambient pressure and $T_{c}=630 \mathrm{~K}$. The continuous linear decrease of the SHG intensity that disappears at $7 \mathrm{GPa}$ and the existence of a Raman active mode at $244 \mathrm{~cm}^{-1}$ that first softens up to $7 \mathrm{GPa}$ and then hardens with pressure are used to discuss the nature of the phase transition of $\mathrm{CaMnTi}_{2} \mathrm{O}_{6}$, for which a $d T_{c} / d P=-48 \mathrm{~K} / \mathrm{GPa}$ has been found. Neither a volume contraction nor a change in the normalized pressure on the Eulerian strain is observed across the phase transition with all the unit-cell volume data following a second-order Birch-Murnaghan equation of state with a bulk modulus of $B_{0}=182.95(2) \mathrm{GPa}$.
\end{abstract}

DOI: 10.1103/PhysRevB.96.094101

\section{INTRODUCTION}

Multiferroics have attracted a significant amount of attention as they potentially offer the prospect of doubling the capacity of information storage in combined MRAMFRAM memories [1]. The largest coexisting ferroelectric and magnetic effects are found in type-I multiferroics, in which an ambient-temperature ferroelectric compound containing a magnetic ion shows an antiferromagnetic order at sufficiently low temperature. Unfortunately, while most type-I multiferroics have a large spontaneous polarization, they do not show bulk ferroelectricity due to their large leakage and coercive field [2]. Recently, Aimi et al. [3] have discovered that $\mathrm{CaMnTi}_{2} \mathrm{O}_{6}$ is a multiferroic with a moderate spontaneous polarization of $\sim 24 \mu \mathrm{C} / \mathrm{cm}^{2}$ and the first example of an oxide containing $\mathrm{Mn}^{2+}$ allowing a polarization reversal at ambient temperature, thus leading to a novel class of type-I multiferroics.

$\mathrm{CaMnTi}_{2} \mathrm{O}_{6}$ crystallizes in a tetragonal A-site-orderedtype double-perovskite structure [3] (Fig. 1), very similar to centrosymmetric $\mathrm{CaFeTi}_{2} \mathrm{O}_{6}$. In this structure, the $\mathrm{Ca}^{2+}$ ions are tenfold coordinated, $\mathrm{Ti}^{4+}$ occupy tilted octahedra, one half of the $\mathrm{Mn}^{2+}$ are tetrahedrally coordinated (Mn1), and the other half are in a pseudosquare planar configuration (Mn2). However, in contrast to $\mathrm{CaFeTi}_{2} \mathrm{O}_{6}$, the square-planar $\mathrm{Mn}^{2+}$ and the octahedrally coordinated $\mathrm{Ti}^{4+}$ are shifted along the $c$ axis in $\mathrm{CaMnTi}_{2} \mathrm{O}_{6}$. This breaks the center of inversion, lowering the symmetry from space group $\mathrm{P}_{2} / \mathrm{nmc}$ observed in $\mathrm{CaFeTi}_{2} \mathrm{O}_{6}$ to $P 4_{2} m c$ and generates a spontaneous

\footnotetext{
*javier.ruiz-fuertes@uv.es
}

polarization. Tetrahedrally coordinated $\mathrm{Mn}^{2+}$ and the $\mathrm{Ca}^{2+}$ ions play almost no role in the ferroelectricity of $\mathrm{CaMnTi}_{2} \mathrm{O}_{6}$. Aimi et al. [3] have shown that with increasing temperature the off-centering of the square-planar coordinated $\mathrm{Mn}^{2+}$ and of the $\mathrm{Ti}^{4+}$ decreases. Concomitantly, the second-harmonicgeneration (SHG) signal decreases monotonically with temperature up to the Curie temperature $T_{c}=630 \mathrm{~K}$, where it becomes zero due to the ferroelectric (space group $\mathrm{P} 4_{2} \mathrm{mc}$ ) to paraelectric (space group $P 4_{2} / n m c$ ) phase transition. In paraelectric $\mathrm{CaMnTi}_{2} \mathrm{O}_{6}$ the $\mathrm{Ti}^{4+}$ ions move to the center of their octahedra but square-planar $\mathrm{Mn}^{2+}$ keep their shift along $c$, with one half of the $\mathrm{Mn}^{2+}$ ions shifting up and the other half shifting down [Fig. 1(c)]. Thus, overall, the total spontaneous polarization disappears, and a center of inversion emerges in space group $P 4_{2} / n m c$.

In the present work we study the nature of the ferroelectric to paraelectric phase transition of $\mathrm{CaMnTi}_{2} \mathrm{O}_{6}$ by inducing it under high pressure. We perform SHG and Raman spectroscopic measurements to find the pressure-induced phase transition, understand the effect of pressure on the spontaneous polarization, and investigate the behavior of the Raman active modes. Finally, the high-pressure paraelectric structure of $\mathrm{CaMnTi}_{2} \mathrm{O}_{6}$ is solved by $\mathrm{x}$-ray diffraction (XRD).

\section{EXPERIMENTAL DETAILS}

$\mathrm{CaMnTi}_{2} \mathrm{O}_{6}$ was synthesized using pure ilmenite-type $\mathrm{MnTiO}_{3}$ and $\mathrm{CaTiO}_{3}$ sealed inside a platinum capsule at $7 \mathrm{GPa}$ and $1700{ }^{\circ} \mathrm{C}$ in a multianvil press for $30 \mathrm{~min}$ following the work by Aimi et al. [3]. The multianvil experiment was performed at GFZ Potsdam with a 18/11 assembly, which was calibrated at room temperature against the phase transitions in $\mathrm{Bi}$ 


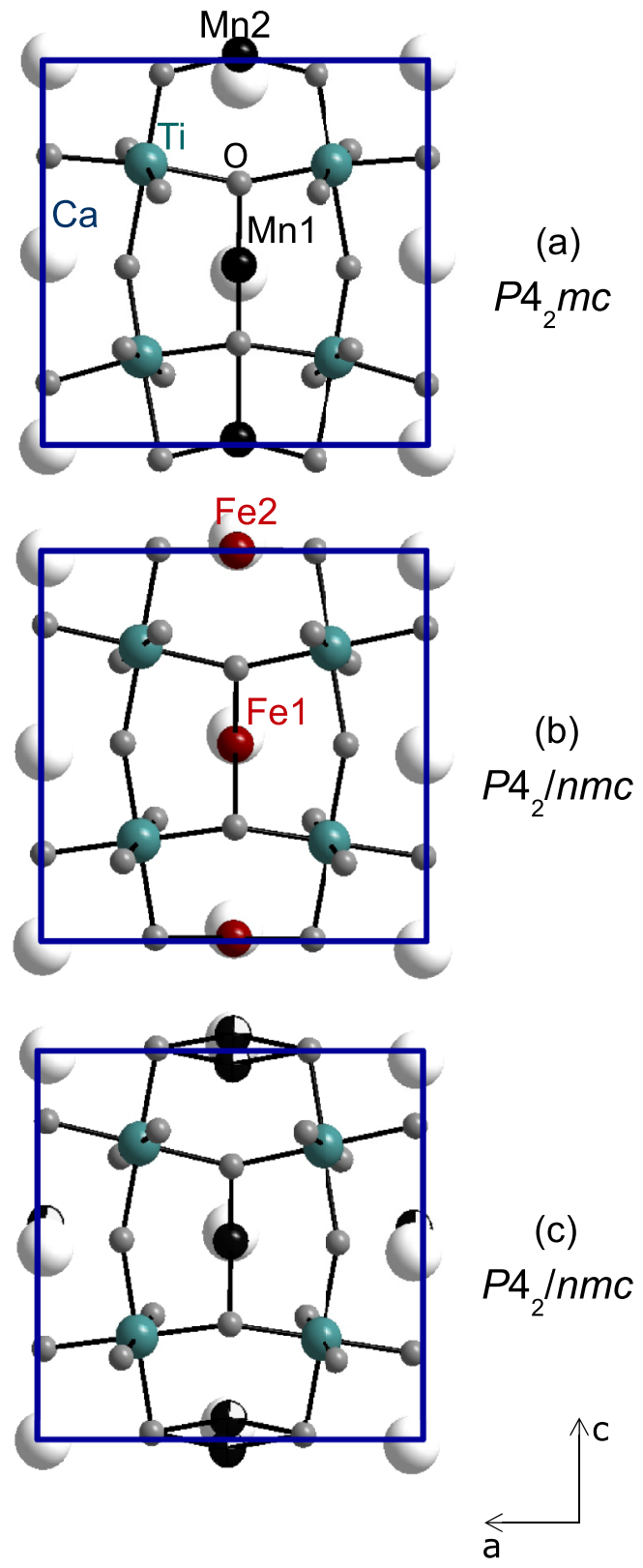

FIG. 1. Projection of the A-site-ordered perovskite structure projected along the [010] direction for (a) the ferroelectric $\mathrm{CaMnTi}_{2} \mathrm{O}_{6}$ (space group $P 4_{2} m c$ ), (b) $\mathrm{CaFeTi}_{2} \mathrm{O}_{6}$ (space group $P 4_{2} / n m c$ ), and (c) paraelectric $\mathrm{CaMnTi}_{2} \mathrm{O}_{6}$ (space group $P 4_{2} / n m c$ ).

metal $[4,5]$. Calibrations at high temperature are based on the following phase transitions: , garnet-perovskite for $\mathrm{CaGeO}_{3}$ [6] and coesite-stishovite for $\mathrm{SiO}_{2}$ [7]. Stepped graphite heaters were employed, and temperatures were measured with type-C thermocouples. We obtained orange single crystals $10-50 \mu \mathrm{m}$ in size embedded in powder.

High-pressure SHG, Raman spectroscopy, and singlecrystal XRD (SXRD) experiments were carried out using Boehler-Almax diamond-anvil cells (DACs) [8] equipped with diamonds with $350-\mu \mathrm{m}$ culets and tungsten gaskets, indented to a thickness of $40 \mu \mathrm{m}$. Holes with a diameter of $150 \mu \mathrm{m}$ served as sample chambers. For the Raman spectroscopy and SXRD experiments single crystals with a thickness of $\sim 10 \mu \mathrm{m}$ were employed. For the SHG runs we used either a single crystal or powder pellets. SHG signals of acentric quartz and centrosymmetric $\mathrm{Al}_{2} \mathrm{O}_{3}$ were used as a reference to calibrate the baseline of the SHG measurements. In all these experiments we placed the samples inside the gasket hole together with a ruby chip to measure the pressure [9] and $\mathrm{Ne}$ as pressure-transmitting medium. The SHG intensities at $\lambda_{2 \omega}=527 \mathrm{~nm}$ were measured in transmission geometry using the setup described by Bayarjargal et al. [10]. The Raman experiment was performed in the backscattering configuration with a Renishaw (RM-1000) spectrometer equipped with a grating with 1800 grooves $/ \mathrm{mm}$ and a spectral resolution of around $2 \mathrm{~cm}^{-1}$. The excitation source was a $\mathrm{HeNe}$ laser $(\lambda=633 \mathrm{~nm})$ focused down to a $10-\mu \mathrm{m}$ spot with a $20 \times$ long working distance objective. An edge filter was used to filter the laser, allowing measurements above $130 \mathrm{~cm}^{-1}$. The SXRD experiment was performed at the Extreme Conditions beamline at PETRA III with a wavelength of $\lambda=0.29036 \AA$ focused down to $3 \times 8 \mu \mathrm{m}^{2}$ (FWHM) with compound refractive lens (CRL) mirrors and using a PerkinElmer detector placed $415.5 \mathrm{~mm}$ from the sample. The diffraction images were collected by $0.5^{\circ} \omega$ scanning. The image format was converted according to the procedure described by Rothkirch et al. [11] for further processing with the CRYSALIS ${ }^{\text {Pro }}$ software [12] for indexing reflections and intensity data reduction. Crystal structures at 0.8, 2.0, 5.6, 7.0, 9.0, 11.5, 12.7, and 14.3 GPa were solved with the Patterson method implemented in SHELXS97-2 and refined with SHELXL97-2 [13].

For the synchrotron powder XRD (PXRD) experiments we employed gas-membrane-driven DACs equipped with $300-\mu \mathrm{m}$ culet diamonds and Inconel gaskets indented to $35 \mu \mathrm{m}$ in thickness with holes of $100 \mu \mathrm{m}$. Powder pellets were loaded together with copper as a pressure gauge [14], and a mixture of 4:1 methanol-ethanol was the pressure-transmitting medium. These experiments were carried out at the Materials Science and Powder Diffraction (MSPD) beamline at the ALBA-CELLS synchrotron $(\lambda=0.4246 \AA)$ up to $5 \mathrm{GPa}$. The beam was focused down to $20 \times 20 \mu \mathrm{m}^{2}$ (FWHM) with Kirkpatrick-Baez mirrors, and a Rayonix CCD detector was placed $300 \mathrm{~mm}$ from the sample. FIT2D [15] was used to integrate the diffraction patterns, and GSAS $[16,17]$ was employed to carry out the Le Bail refinements [18]. For the Le Bail refinements, a pseudo-Voigt function was employed according to Thompson et al. [19] in conjunction with an asymmetry correction given by Finger et al. [20].

\section{RESULTS}

\section{A. Second-harmonic generation}

The pressure dependence of the SHG signal $I_{2 \omega}$ is shown in Fig. 2. With pressure, the SHG signal decreases linearly from an initial value of $\sim 1000$ counts up to $7 \mathrm{GPa}$ when, within our experimental accuracy, no signal can be detected anymore. We interpret this as the ferroelectric phase transition of $\mathrm{CaMnTi}_{2} \mathrm{O}_{6}$.

Hence, $\mathrm{CaMnTi}_{2} \mathrm{O}_{6}$ undergoes a ferroelectric to paraelectric phase transition either at ambient pressure [3] and $T_{c}=$ $630 \mathrm{~K}$ or at ambient temperature and $P_{c}=7 \mathrm{GPa}$. This result associated with second-order phase transitions in ferroelectrics 


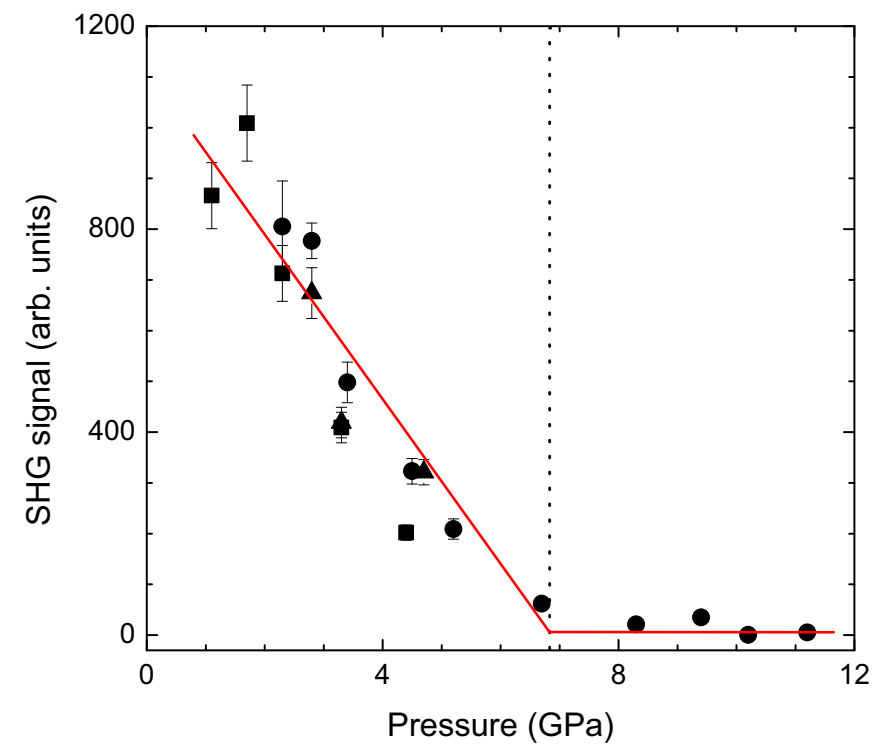

FIG. 2. Pressure dependence of the second-harmonic-generation signal showing a continuous decrease up to $\sim 7 \mathrm{GPa}$. Different symbols correspond to different experiments. The solid circles are data from the single-crystal experiment, whereas the solid squares and triangles correspond to powder data. The vertical dashed line marks the phase boundary, and the red solid lines are guides for the eye.

[21] indicates that the Curie temperature of $\mathrm{CaMnTi}_{2} \mathrm{O}_{6}$ decreases with pressure at $d T_{c} / d P=-48 \mathrm{~K} / \mathrm{GPa}$, similar to the value of $-52 \mathrm{~K} / \mathrm{GPa}$ observed for $\mathrm{BaTiO}_{3}$ [21]. With the objective of investigating the behavior of the optical modes at the zone center and in order to solve the high-pressure structure of $\mathrm{CaMnTi}_{2} \mathrm{O}_{6}$, we present in the following sections the results of high-pressure Raman spectroscopy and XRD studies.

\section{B. Raman spectroscopy}

The A-site-ordered-type structure of $\mathrm{CaMnTi}_{2} \mathrm{O}_{6}$ with point group $4 \mathrm{~mm}$ has 88 vibrational zone-center modes. Two of them, with irreducible representations $A_{1}+E$, correspond to acoustic phonons, and the ten $A_{2}$ modes are silent. The remaining optic phonons $\left(17 A_{1}+18 B_{1}+10 B_{2}+31 E\right)$ are Raman active, with the $A_{1}$ and $E$ modes being polar and therefore also IR active. Given the number of Raman active modes and the fact that 41 of them are polar and therefore will show longitudinal optical-transverse optical (LO-TO) splitting, one can expect a Raman spectrum with a continuous character, with a significant overlap of Raman bands and few distinct features. A selection of Raman spectra of $\mathrm{CaMnTi}_{2} \mathrm{O}_{6}$ at different pressures is shown in Fig. 3. Although an unambiguous Raman assignment has not been possible because the size of the crystals $(<50 \mu \mathrm{m})$ has not allowed us to orient them, we can tentatively assign the most intense bands.

Although $\mathrm{CaMnTi}_{2} \mathrm{O}_{6}$ has almost six times more Raman active modes than polar $\mathrm{ZnTiO}_{3}$ [22] in the $\mathrm{LiNbO}_{3}$-type structure, the Raman spectra of these two compounds mutually resemble each other. This is reasonable if we consider that the polar crystal structures of both $\mathrm{CaMnTi}_{2} \mathrm{O}_{6}$ and $\mathrm{ZnTiO}_{3}$ are

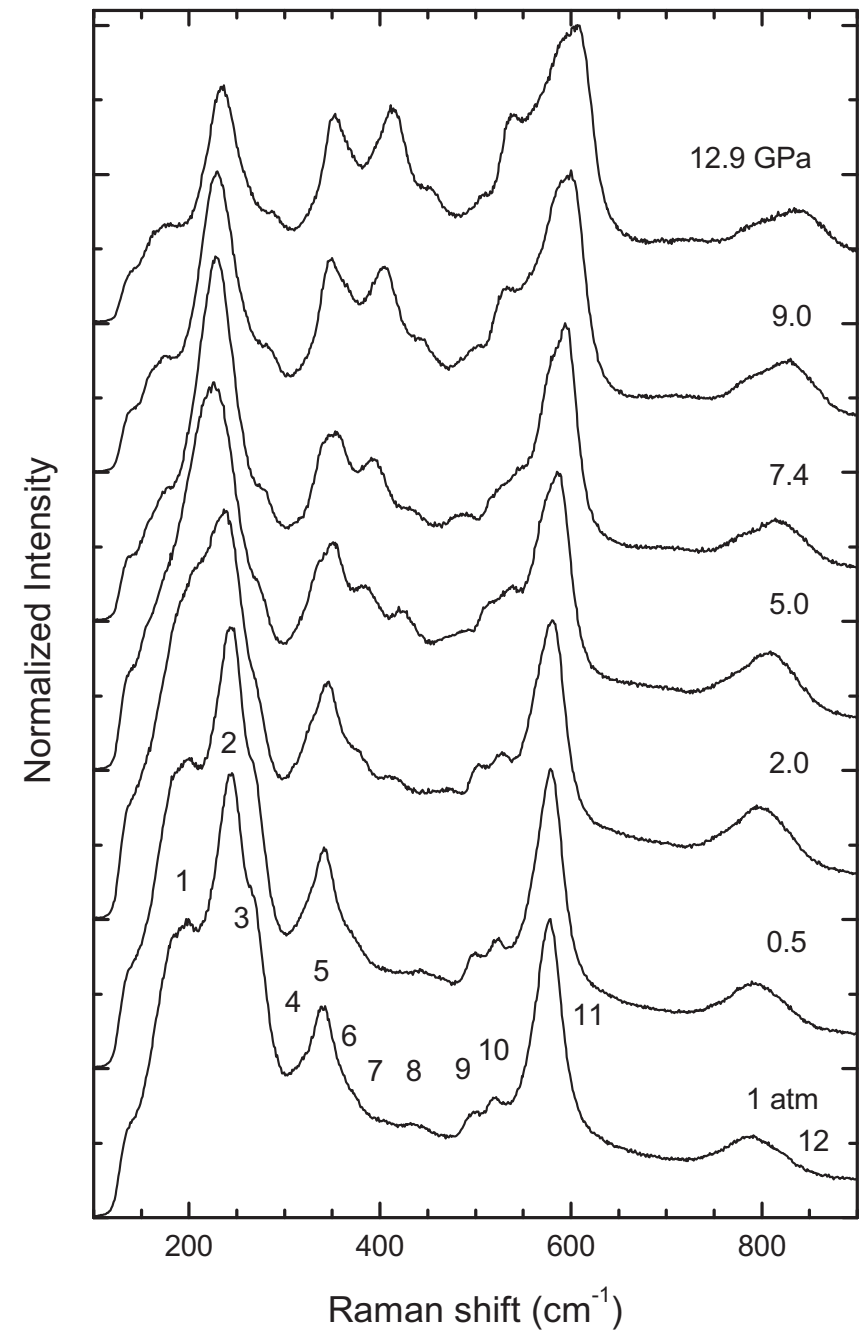

FIG. 3. Raman spectra at different pressures up to $12.9 \mathrm{GPa}$. The numbers shown in the spectrum at $1 \mathrm{~atm}$ denote the labeling of the observed Raman modes.

related to the perovskite structure. In the case of $\mathrm{CaMnTi}_{2} \mathrm{O}_{6}$, the unit-cell edge is doubled in conjunction with a distortion of the $\mathrm{TiO}_{6}$ octahedra to form the polar, tetragonal structure in $4 \mathrm{~mm}$, while a distortion of the perovskite structure according to the $\mathrm{LiNbO}_{3}$ structure leads to the polar, trigonal crystal class $3 \mathrm{~m}$.

Similar to $\mathrm{ZnTiO}_{3}, \mathrm{CaMnTi}_{2} \mathrm{O}_{6}$ also shows two intense bands located at around $244 \mathrm{~cm}^{-1}\left(v_{2}\right)$ and $590 \mathrm{~cm}^{-1}\left(v_{11}\right)$ and an isolated broad band at around $800 \mathrm{~cm}^{-1}\left(v_{12}\right)$. In polar $\mathrm{ZnTiO}_{3}[22,23] \nu_{2}$ is an $A_{1}$ mode polarized along the $c$ direction and consists of an antiphase vibration of the $\mathrm{Ti}^{4+}$ ion against the octahedral oxygen framework with the $\mathrm{Zn}^{2+}$ ion at rest; $v_{11}$ is another $A_{1}$ mode that consists of a rocking mode of the oxygen octahedral framework with $\mathrm{Ti}^{4+}$ and $\mathrm{Zn}^{2+}$ at rest, and $v_{12}$ is the LO mode of $v_{11}$. As $v_{2}$ and $v_{11}$ are pure $\mathrm{TiO}_{6}$ modes, it is reasonable to assume that they have a similar eigenvector in both compounds, and therefore, we tentatively assign them to $A_{1}$ modes in $\mathrm{CaMnTi}_{2} \mathrm{O}_{6}$ as well. This hypothesis is further supported under high pressure. While 11 of the 12 observed modes shift to higher energies with increasing pressure, the $v_{2}$ mode softens up to around $7 \mathrm{GPa}$ 
(Fig. 3) when it overlaps with the mode at lower-frequency $v_{1}$ and starts hardening. Above 7.4 GPa other bands change their intensity or vanish, and additional bands appear. We interpret these changes in the Raman spectrum of $\mathrm{CaMnTi}_{2} \mathrm{O}_{6}$ at $7.4 \mathrm{GPa}$ as the onset of the phase transition. This transition pressure is in good agreement with the transition pressure deduced from the disappearance of the SHG signal described above.

Different from $\mathrm{CaMnTi}_{2} \mathrm{O}_{6}$, in polar $\mathrm{ZnTiO}_{3}$ [22] or $\mathrm{MnTiO}_{3}$ [24] the $v_{2}\left[A_{1}(2)\right]$ Raman mode vanishes in the first-order phase transition, while in $\mathrm{CaMnTi}_{2} \mathrm{O}_{6}$ it stays and hardens as an indication of a less abrupt phase transformation. In fact, the behavior observed in $\mathrm{CaMnTi}_{2} \mathrm{O}_{6}$ was previously reported in other pressure-induced second-order phase transitions [25] and indicates that even though the mode softening up to the phase transition is not driving the phase transition, it is sensitive to the motion of the ions involved in the transition process. This indicates that most likely the $v_{2}$ mode softens up to the phase transition, and once the $\mathrm{Ti}^{4+}$ ion falls in the centrosymmetric site, the condition to introduce the center of inversion in the structure, the same mode starts hardening with pressure. Although this hypothesis will be reinforced in the next section, such behavior can be explained as follows. Each Ti atom shares one oxygen atom with a Mn2. Under pressure the Mn2-O bond with respect to the $a b$ plane gets closer to $180^{\circ}$, which should increase the frequency of the mode. However, the hardening is compensated below the phase transition by the softening of the mode produced by the fact that the $\mathrm{Ti}$ atoms are getting closer to the centrosymmetric position, and therefore, the vibration of the $\mathrm{Ti}$ atoms is facilitated. Once the Ti atoms occupy the center of inversion in the phase transition, their oscillation along $c$ reduces, and the hardening due to the reduction of the Mn2-O angle with respect to the $a b$ plane takes over dominating the process.

Figure 4 shows the frequencies of the observed Raman active modes up to $12.9 \mathrm{GPa}$. As stated before, we observe that the frequencies of all but two modes in the low-pressure phase and all modes in the high-pressure phase increase with increasing pressure, while the frequency of the $v_{2}$ mode decreases until the onset of the phase transition. The pressure coefficients, $a_{i}=d \omega_{i} / d P$, obtained from linear fits, are shown in Table I together with their Grüneisen parameters $\gamma_{i}=B_{0} / \omega a_{i}$.

\section{X-ray diffraction}

The pressure dependences of the unit-cell volume and of the $a / c$ ratio, as well as the dependence of the normalized pressure $F$ on the Eulerian strain $f$ of $\mathrm{CaMnTi}_{2} \mathrm{O}_{6}$, are shown in Fig. 5 for the PXRD and the SXRD experiments up to 5 and $14.3 \mathrm{GPa}$, respectively. We do not observe, within the resolution of our experiments, any indication of a phase transition up to the maximum pressure reached (14.3 $\mathrm{GPa})$. The $a / c$ ratio of the lattice parameters shows an increasing symmetrization of the metric with pressure. The dependence of the normalized pressure $F$ on the Eulerian strain $f$ often is a good indicator for a structural phase transition. Here, while there is significant scatter in our data, it seems that the best fit yields $d F / d f=0$, and therefore, all $P-V$ data up to 14.3 GPa are well described with a single second-order Birch



FIG. 4. Raman shifts of $\mathrm{CaMnTi}_{2} \mathrm{O}_{6}$ as a function of pressure measured during compression. The vertical dotted line marks the suggested phase-transition boundary. The solid and dotted blue straight lines are linear fits to the data. The red solid lines mark the linear fit to the two modes at low frequency, including the soft mode $v_{2}$. Modes are labeled according to Fig. 3.

Murnaghan equation of state. This is in contrast to the results obtained from the SHG experiments and Raman spectroscopy discussed in previous sections that indicate the occurrence of a phase transition at $\sim 7 \mathrm{GPa}$.

As we explained in the Introduction (Fig. 1), the structure of ferroelectric $\mathrm{CaMnTi}_{2} \mathrm{O}_{6}$ is described in the noncentrosymmetric space group $\mathrm{P}_{2} \mathrm{mc}$; however, this structure is very similar to the structure of $\mathrm{CaFeTi}_{2} \mathrm{O}_{6}$ with the centrosymmetric space group $P 4_{2} / n m c$. In the eight SXRD experiments performed at different pressures we did not find enough systematic extinctions to distinguish between tetragonal space groups $P 4_{2}, P 4_{2} m c$, and $P 4_{2} / n m c$. Space group $P 4_{2}$ is a translationengleiche subgroup of $P 4_{2} m c$, which is simultaneously a translationengleiche subgroup of $P 4_{2} / n m c$. In order to avoid any artificial symmetry constraints, we initially solved the structure of $\mathrm{CaMnTi}_{2} \mathrm{O}_{6}$ at different pressures in space group $P 4_{2}$. We found that the structure in $P 4_{2}$ remained identical to the structure solved by Aimi et al. [3] in space group $P 4_{2} m c$ up to $14.3 \mathrm{GPa}$. Then we used the program PLATON [26] to search for higher symmetry. We found that the symmetry of the structure was better described in $P 4_{2} m c$ up to $7 \mathrm{GPa}$ as 
TABLE I. Phonon frequencies of $\mathrm{CaMnTi}_{2} \mathrm{O}_{6}$ in the low-pressure ferroelectric and high-pressure paraelectric phases. The pressure coefficients $a_{i}$ of the modes and their Grüneisen parameters $\gamma_{i}$ are calculated considering the bulk modulus $B_{0}=182.95(2) \mathrm{GPa}$ obtained from single-crystal XRD for both phases. Modes are numbered with increasing frequency. In the paraelectric phase those modes that do not have a correspondence in the ferroelectric phase are marked with a prime.

\begin{tabular}{|c|c|c|c|c|c|c|c|}
\hline & Ferroe & ric at $1 \mathrm{~atm}$ & & & araelect & tric at $7.4 \mathrm{GPa}$ & \\
\hline Mode & $\begin{array}{c}\omega \\
\left(\mathrm{cm}^{-1}\right.\end{array}$ & $\begin{array}{c}a_{i} \\
\left.\mathrm{~m}^{-1} / \mathrm{GPa}\right)\end{array}$ & $\gamma$ & Mode & $\begin{array}{c}\omega \\
\left(\mathrm{cm}^{-1}\right)\end{array}$ & $\begin{array}{c}a_{i} \\
\left(\mathrm{~cm}^{-1} / \mathrm{GPa}\right)\end{array}$ & $\gamma$ \\
\hline$v_{1}$ & 202 & 2.7 & 2.45 & $v_{2}$ & 228 & 2.1 & 1.69 \\
\hline$v_{2}$ & 244 & -3.5 & -2.62 & $v_{3}^{\prime}$ & 279 & 3.6 & 2.36 \\
\hline$v_{3}$ & 272 & 0.8 & 0.56 & $v_{4}$ & 344 & 2.4 & 1.38 \\
\hline$v_{4}$ & 322 & 2.4 & 1.38 & $v_{6}$ & 395 & 3.3 & 1.62 \\
\hline$v_{5}$ & 341 & 2.1 & 1.13 & $v_{7}$ & 432 & 3.6 & 1.61 \\
\hline$v_{6}$ & 372 & 3.3 & 1.62 & $v_{8}$ & 483 & 3.9 & 1.56 \\
\hline$v_{7}$ & 408 & 3.6 & 1.61 & $v_{9}^{\prime}$ & 529 & 1.7 & 0.59 \\
\hline$v_{8}$ & 456 & 3.9 & 1.56 & $v_{11}$ & 595 & 2.3 & 0.73 \\
\hline$\nu_{9}$ & 498 & 3.3 & 1.21 & $v_{12}$ & 818 & 3.7 & 0.86 \\
\hline$v_{10}$ & 520 & 3.1 & 1.09 & & & & \\
\hline$v_{11}$ & 578 & 2.3 & 0.73 & & & & \\
\hline$v_{12}$ & 791 & 3.7 & 0.86 & & & & \\
\hline
\end{tabular}
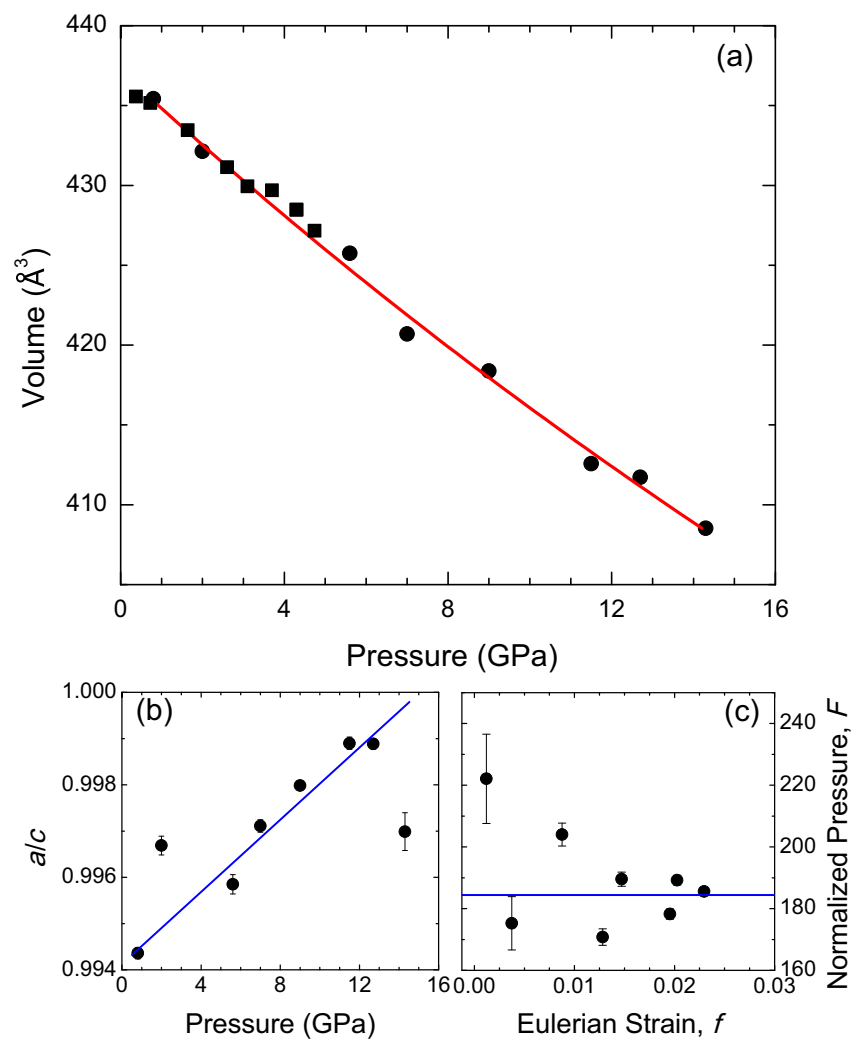

FIG. 5. (a) Pressure dependence of the unit-cell volume of $\mathrm{CaMnTi}_{2} \mathrm{O}_{6}$. The solid line denotes the fit to a second-order BirchMurnaghan equation of state. (b) Pressure dependence of the $a / c$ ratio showing a monotonic increase. The solid line is a guide for the eye. (c) Relation between the Eulerian strain $f$ and the normalized pressure $F$. Data obtained from the powder diffraction experiment are represented by squares, and the single-crystal data are shown by circles.



FIG. 6. Pressure dependence of the anisotropic $U_{33}$ thermal displacement parameter of $\mathrm{Mn}^{2+}$ in the $\mathrm{Mn} 2$ position for the ferroelectric (space group $\mathrm{P}_{2} \mathrm{mc}$ ) up to $7 \mathrm{GPa}$ and the paraelectric (space group $P 4_{2} m c$ ) structures of $\mathrm{CaMnTi}_{2} \mathrm{O}_{6}$ considering no splitting. Solid lines are guides for the eye.

Aimi et al. [3] had shown at ambient temperature and pressure. However, from 9 to $14.3 \mathrm{GPa}$ we found that the symmetry was higher and the structure was better described in space group $P 4_{2} / n m c$, confirming the occurrence of a ferroelectric to paraelectric phase transition as deduced from the SHG experiments before. Using the transformation provided by PLATON, we refined the structures from 0.8 to $7 \mathrm{GPa}$ in space group $\mathrm{P}_{2} \mathrm{mc}$ and from 9 to $14.3 \mathrm{GPa}$ in $P 4_{2} / \mathrm{nmc}$. We found that the paraelectric structure was identical to the structure of $\mathrm{CaFeTi}_{2} \mathrm{O}_{6}$, i.e., with no off-center shift along $c$ for Ti and Mn2. However, the $U_{33}$ term of the anisotropic thermal displacement matrix for Mn2 increased significantly across the phase transition. The pressure evolution of the $\mathrm{Mn} 2 U_{33}$ is shown in Fig. 6.

We found that up to $7 \mathrm{GPa} U_{33}$ takes a constant value of $0.02 \AA^{2}$, reasonably low considering that these are highpressure data. However, from $9 \mathrm{GPa}$ when the structure transforms to $P 4_{2} / n m c$ and the Mn2 atoms are constrained by symmetry to stay in the center of the polyhedron [Fig. 1(b)], the value of their $U_{33}$ term abruptly increases to $0.12 \AA^{2}$ (Fig. 6). This behavior is not observed for the Mn1 or any of the other atoms and is indicative of a large positional disorder of Mn2 along $c$. At high temperature and ambient pressure, Aimi et al. [3] showed that the structure of paraelectric $\mathrm{CaMnTi}_{2} \mathrm{O}_{6}$ at $700 \mathrm{~K}$, although described in space group $P 4_{2} / n m c$, has $50 \%$ of the Mn2 shifted up along $c$ and the other $50 \%$ shifted down. When we refine the structure of $\mathrm{CaMnTi}_{2} \mathrm{O}_{6}$ with a split-atom position for $\mathrm{Mn} 2$ with an occupancy of $50 \%$, we confirm that the pressure-induced ferroelectric transformation at ambient temperature is identical to the temperature-induced transformation at ambient pressure [27].

Although a temperature increase causes an expansion [3] of the lattice with $\sim 2.74 \times 10^{-5} \mathrm{~K}^{-1}$ and pressure compresses the lattice, both increasing pressure and increasing temperature lead to a reduction of the Mn2 and Ti off-center shift along 

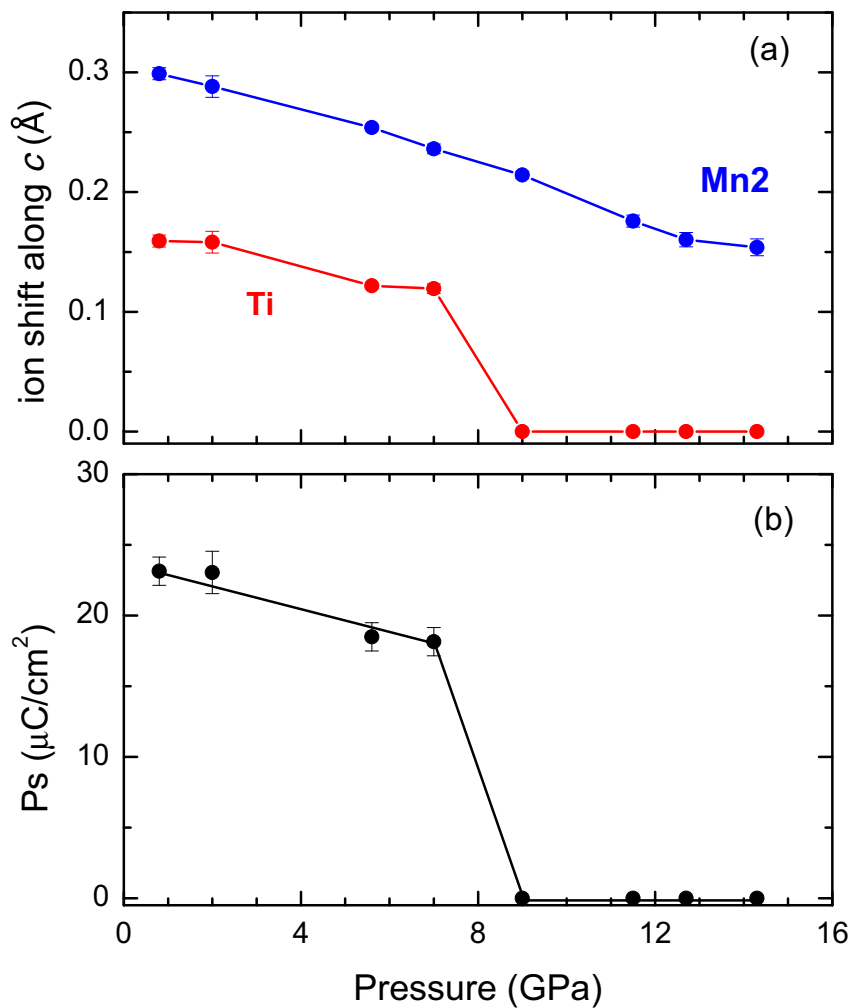

FIG. 7. (a) Off-center shifts along $c$ for Ti and Mn2 as a function of pressure for $\mathrm{CaMnTi}_{2} \mathrm{O}_{6}$ obtained from our SXRD experiments. (b) Pressure dependence of the spontaneous polarization $P_{s}$ estimated according to the point-charge model $P_{s}=\left(\sum_{i} q_{i} \delta_{i}\right) / V$, where $q_{i}$ is the nominal charge of atom $i$ and $\delta_{i}$ is the shift of atom $i$ along $c$.

$c$ and therefore to a reduction of the spontaneous polarization $P_{s}$ (Fig. 7). Such an identical response to both pressure and temperature increases is the result of a phase transition driven only by temperature.

Our result shows that the phase transition of $\mathrm{CaMnTi}_{2} \mathrm{O}_{6}$ is entirely driven by the off-centering of the $\mathrm{Ti}^{4+}$ ions. There are four chemical formula units per unit cell, and hence, there are eight $\mathrm{Ti}^{4+}$ ions and only two $\mathrm{Mn}^{2+}$ ions on the Mn2 site. Thus, when the shift of the $\mathrm{Ti}^{4+}$ ion gets close enough to the centrosymmetric position, i.e., a shift along $c$ of $\sim 0.1 \AA$ (Fig. 6), the phase transition occurs, forcing the $\mathrm{Mn}^{2+}$ ion in the Mn2 site (still with a shift of $\sim 0.2 \AA$ ) to quench its shift, resulting in a disordered Mn2 position, with the Mn2 keeping the distortion.

\section{CONCLUSIONS}

With a combined pressure-dependent study of the secondharmonic-generation signal intensity, Raman spectroscopy, and single-crystal and powder x-ray diffraction, we have shown that $\mathrm{CaMnTi}_{2} \mathrm{O}_{6}$ undergoes a phase transition at $\sim 7 \mathrm{GPa}$ and ambient temperature to the same paraelectric structure observed above $630 \mathrm{~K}$ and ambient pressure. This has allowed us to determine that the Curie temperature decreases with pressure at $d T_{c} / d P=-48 \mathrm{~K} / \mathrm{GPa}$. We have found with Raman spectroscopy a mode at zone center at $244 \mathrm{~cm}^{-1}$ that softens with pressure. We have tentatively assigned this mode to an antiphase vibration of the $\mathrm{Ti}^{4+}$ ion against its octahedral oxygen framework, indicating that the mode, despite not driving the phase transition, is coupled to the shift of $\mathrm{Ti}^{4+}$. With SXRD we have solved the structure of the high-pressure phase of $\mathrm{CaMnTi}_{2} \mathrm{O}_{6}$ and followed the evolution of both the Ti and Mn2 shifts with pressure. This has allowed us to observe that the phase transition is mostly dominated by the shift of the $\mathrm{Ti}$ atoms, with the $\mathrm{Mn} 2$ atom playing a minor role.

\section{ACKNOWLEDGMENTS}

J.R.-F. thanks the Juan de la Cierva Program (IJCI-201420513) of the Spanish MINECO and D. Errandonea and A. Segura for fruitful discussions. N.S. acknowledges the DFG, Germany (Project No. RA2585/1-1). M.K.-M. thanks A. Ebert and R. Schulz for the technical support during the MultiAnvil experiments. B.W. acknowledges the BMBF, Germany (Projects No. 05K10RFA and No. 05K13RF1). This paper was partially supported by the Spanish Ministerio de Economía y Competitividad (MINECO) under Grants No. MAT201346649-C04-01/03-P, No. MAT2016-75586-C4-1/3-P, and No. MAT2015-71070-REDC (MALTA Consolider). The authors thank synchrotron ALBA-CELLS (Project No. 2016021588) for beam time allocation at the MSPD line. Parts of this research were carried out at the light source PETRA III at DESY (Project No. I-20160082 EC), a member of the Helmholtz Association (HGF).
[1] S. W. Cheong and M. Mostovoy, Nat. Mater. 6, 13 (2007).

[2] A. Aimi, T. Katsumata, D. Mori, D. S. Fu, M. Itoh, T. Kyômen, K. Hiraki, T. Takahashi, and Y. Inaguma, Inorg. Chem. 50, 6392 (2011).

[3] A. Aimi, D. Mori, K. Hiraki, T. Takahashi, Y. J. Shan, Y. Shikaro, J. Zhou, and Y. Inaguma, Chem. Mater. 26, 2601 (2014).

[4] E. C. Lloyd, NBS Spec. Publ. (US) 326, 1 (1971).

[5] G. J. Piermarini and S. Block, Rev. Sci. Instrum. 46, 973 (1975).

[6] J. Susaki, M. Akaogi, S. Akimoto, and O. Shimoura, Geophys. Res. Lett. 12, 729 (1985).

[7] M. Akaogi, H. Yusa, K. Shiraishi, and T. Suzuki, J. Geophys. Res. 100, 22337 (1995).
[8] R. Boehler, Rev. Sci. Instrum. 77, 115103 (2006).

[9] H. K. Mao, P. M. Bell, J. W. Shaner, and D. J. Steinberg, J. Appl. Phys. 49, 3276 (1978).

[10] L. Bayarjargal, B. Winkler, E. Haussühl, and R. Boehler, Appl. Phys. Lett. 95, 061907 (2009).

[11] A. Rothkirch, G. D. Gatta, M. Meyer, S. Merkel, M. Merlini, and H. P. Liermann, J. Synchrotron Radiat. 20, 711 (2013).

[12] Agilent, CRYSALIS ${ }^{\text {Pro }}$ software system, version 1.171.36.28, Agilent Technologies UK Ltd., Oxford, UK, 2013.

[13] G. M. Sheldrick, Acta Crystallogr., Sect. A 64, 112 (2008).

[14] Y. Wang, J. Zhang, H. Xu, Z. Lin, L. L. Daemen, Y. Zhao, and L. Wang, Appl. Phys. Lett. 94, 071904 (2009). 
[15] A. P. Hammersley, S. O. Svensson, M. Hanfland, A. N. Fitch, and D. Häusermann, High Pressure Res. 14, 235 (1996).

[16] A. C. Larson and R. B. Von Dreele, Los Alamos National Laboratory, Report No. LAUR 87-748 (2004).

[17] B. H. Toby, J. Appl. Crystallogr. 34, 210 (2001).

[18] A. Le Bail, Powder Diffr. 20, 316 (2005).

[19] P. Thompson, D. E. Cox, and J. B. Hastings, J. Appl. Crystallogr. 20, 79 (1987).

[20] L. W. Finger, D. E. Cox, and A. P. Jephcoat, J. Appl. Crystallogr. 27, 892 (1994).

[21] G. A. Samara, Ferroelectrics 2, 277 (1971).

[22] J. Ruiz-Fuertes, B. Winkler, T. Bernert, L. Bayarjargal, W. Morgenroth, M. Koch-Müller, K. Refson, V. Milman, and N. Tamura, Phys. Rev. B 91, 214110 (2015).
[23] V. Caciuc, A. V. Postnikov, and G. Borstel, Phys. Rev. B 61, 8806 (2000).

[24] X. Wu, S. Qin, and L. Dubrovinsky, Geosci. Front. 2, 107 (2011).

[25] D. Errandonea, D. Martínez-García, A. Segura, J. Haines, E. Machado-Charry, E. Canadell, J. C. Chervin, and A. Chevy, Phys. Rev. B 77, 045208 (2008).

[26] A. L. Spek, Acta Crystallogr. D 65, 148 (2009).

[27] See Supplemental Material at http://link.aps.org/supplemental/ 10.1103/PhysRevB.96.094101 to see the atomic coordinates of the refined structures at $0.8,7,9$, and $14.3 \mathrm{GPa}$ together with the data acquisition information and refinement results. The Le Bail refinements of two powder diffractograms are also shown. 